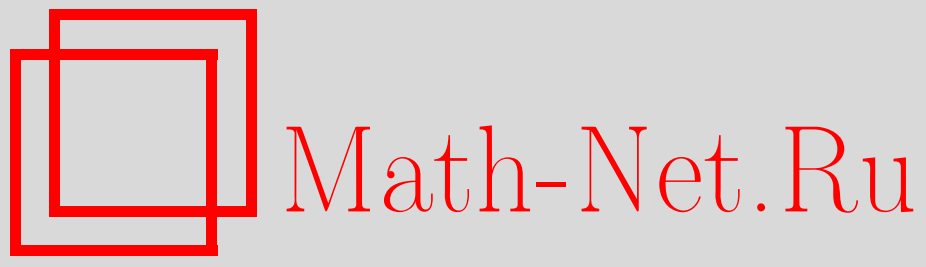

В. И. Арнольд, Оптимизация в среднем и фазовые переходы в управляемых динамических системах, Функи. анализ и его прил., 2002, том 36, выпуск 2, 111

DOI: https://doi.org/10.4213/faa186

Использование Общероссийского математического портала MathNet.Ru подразумевает, что вы прочитали и согласны с пользовательским соглашением

http://www . mathnet.ru/rus/agreement

Параметры загрузки:

IP : 54.172 .240 .79

26 апреля 2023 г., 12:42:18

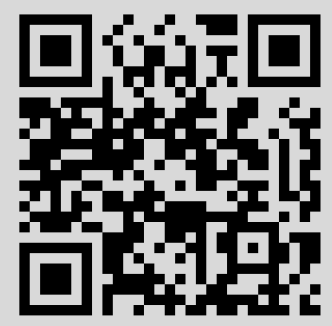


Функциональный анализ и его приложения

2002, т. 36, вып. 2, с. 1-11

УДК 517.938

\title{
Оптимизация в среднем и фазовые переходы в управляемых динамических системах
}

\author{
(с) 2002. В. И. АРнОльд
}

Под оптимизацией в среднем я понимаю максимизацию временно́го среднего значения целевой функции, заданной на фазовом пространстве управляемой динамической системы. Для такой оптимизации иногда следует привести фазовую точку в наилучшее положение равновесия динамической системы, но иногда выгоднее придерживаться совершенно другой стратегии, например, периодически переводить фазовую точку из одного равновесного состояния в другое и обратно. Если управляемая система зависит еще от параметров, то, в зависимости от их значений, самой выгодной может оказаться то одна стратегия, то другая. Из-за этого оптимальное среднее значение, рассматриваемое как функция от параметров, приобретает, вообще говоря, особенности. В настоящей работе явно указаны некоторые простейшие (и оказывающиеся устойчивыми) особенности, возникающие при таких «фазовых переходах» (с изменением характера оптимальной стратегии при изменении параметра) в простейших системах - например, в типичных семействах управляемых систем с одной фазовой и одной управляющей переменными, зависящих от одного параметра, это - разрывы первой или второй производной оптимального среднего значения по параметру.

При бо́льших размерностях (фазового пространства, пространства управляющих переменных и пространства параметров) подобные «фазовые переходы» в семействах общего положения тоже можно исследовать, хотя вычисления быстро становятся сложнее. Исходным пунктом настоящей работы был пример из области управления цементными мельницами [1].

\section{§1. Одномерная система без равновесий}

Фазовым пространством будем для простоты считать окружность $S^{1}$ с координатой $x \bmod 2 \pi$. Уравнение движения управляемой динамической системь это уравнение

$$
\frac{d x}{d t}=v(x, u),
$$

где $u$ - управляющий параметр. Для простоты будем считать его тоже точкой окружности (другой), так что вектор гладкого поля $v$ имеет периоды $2 \pi$ по обеим переменным ${ }^{1)}$.

1) Вместо прямого произведения $\{(x, u)\}$ можно было бы рассматривать расслоение над фазовым пространством или особое подмногообразие пространства касательного расслоения фазового пространства, но я сохраню обозначения (1). 
Пусть $f$ - гладкая цุелевая функциия на фазовой окружности. Мы хотим максимизировать ее временно́е среднее значение,

$$
f_{*}=\lim _{T \rightarrow \infty} \frac{1}{T} \int_{0}^{T} f(x(t)) d t,
$$

проводя максимизацию надлежащим выбором управления, $u=U(t)$ (здесь $x(t)$ - значение решения уравнения (1) при таком выборе управления и при заданном начальном условии, $x(0))$. Эту задачу оптимизации среднего можно исследовать либо при фиксированном начальном условии (от которого будут зависеть и оптимальная стратегия, и экстремальный ответ, $\left.f_{*}\right)$, либо максимизируя среднее значение также и по всевозможным выборам начального условия, $x(0)$.

В этом параграфе мы будем предполагать, что поле $v$ нигде не обращается 8 нуль, и будем заниматься максимизацией среднего значения при не фиксированном начальном условии, т. е. меняя также и $x(0)$ при оптимизации.

Поскольку равновесий нет, фазовая точка за большое время совершает много оборотов вокруг фазовой окружности. Оптимальная стратегия этого движения состоит в том, чтобы двигаться возможно медленнее там, где целевая функция $f$ больше, и возможно быстрее там, где она меньше. Временно́е среднее можно переписать как пространственное среднее вдоль всей фазовой окружности с зависящим от времени весом:

$$
\int_{0}^{T} f(x(t)) d t=\int_{X_{0}}^{X_{1}} f(x) w(x, u) d x,
$$

где $w=1 / v$ (поскольку $d t=d x / v$, согласно (1)). Время движения вдоль орбиты выражается аналогично:

$$
\left.T=\int_{X_{0}}^{X_{1}} w(x, u) d x \quad \text { (для участка орбиты от } X_{0} \text { до } X_{1}\right) .
$$

Функция $w$ всюду положительна. Оптимальную стратегию мы будем искать, выбирая значение параметра управления, $u$, в зависимости от положения фазовой точки, $x$. Таким образом, мы должны распределить некоторую массу на фазовой окружности с плотностью $\rho(x) d x=w(x, u(x)) d x$ так, чтобы максимизировать среднее значение $f_{*}$ целевой функции $f$ по этому распределению $\rho$. Единственное ограничение на выбор распределения состоит в том, что значение $\rho(x)$ в каждой точке должно заключаться между максимумом и минимумом функции $1 / v$ по параметру управления $u$ (при фиксированном $x$ ):

$$
\min _{u} w(x, u)=r(x) \leqslant \rho(x) \leqslant R(x)=\max _{u} w(x, u) .
$$

Если суммарную массу фиксировать, то ясно, что оптимальное распределение таково: для некоторой постоянной $c$ плотность $\rho$ следует выбрать минимальной там, где целевая функция $f$ меньше $c$, и максимальной там, где она больше $c$. Ибо при любом другом распределении часть массы можно было бы перенести, не нарушая ограничения, в область с бо́льшими значениями целевой функции.

От величины суммарной массы $\oint \rho d x$ оптимальное среднее не зависит, так как среднее не меняется при умножении плотности на постоянную. Поэтому достаточно рассмотреть (зависящее от $c$ ) распределение с плотностью

$$
\rho_{c}(x)=r(x) \quad \text { при } f(x)<c, \quad \rho_{c}(x)=R(x) \quad \text { при } f(x)>c,
$$


вычислить с-среднее значение целевой функции $f$ по этому распределению,

$$
f_{c}=\oint f(x) \rho(x) d x / \oint \rho(x) d x,
$$

а затем выбрать постоянную $c$, максимизирующую это $c$-среднее: $f_{*}=\max _{c} f_{c}$.

Для простоты мы предположим, что целевая функция имеет всего две критические точки: невырожденный максимум и невырожденный минимум.

Теорема 1. Oптимальное среднее значение $f_{*}(p)$ усредненной цуелевой функции в зависящем общим образом от параметра р семействе гладких управляемых динамических систем (1) (с перечисленными выше свойствами) является гладкой функцичеи от параметра р, за исключением лищь некоторых дискретно лежащих точек, в окрестности каждой из которых функция $f_{*}$ имеет разрьв первой или второй производной, так что ее график диффеоморфен либо графику функции $|p|$, либо графику функции $p|p|$.

Источником особенностей являются особенности ограничивающих плотность функций $R(x)=\max _{u} w(x, u), r(x)=\min _{u} w(x, u)$. Для управляемых систем $v$ общего положения эти особенности функций максимумов и минимумов диффеоморфны особенностям функций $\pm|x|$. Они происходят от совпадения критических значений в двух точках максимума (минимума).

В типичных однопараметрических семействах функций $v$ встречаются (при определенных исключительных значениях параметра) три более сложные особенности функций максимума и минимума: а) совпадение критических значений, достигаемых в трех разных однотипных критических точках $u_{i}$; b) касание графиков двух конкурирующих функций локального максимума или минимума, достигаемых в двух разных критических точках; с) появление на мгновение «двойного максимума», достигаемого в трехкратной критической точке, как у функции $-u^{4}$, или аналогичное явление для минимумов.

При этих событиях на графике функции максимума или минимума происходят такие перестройки. В случае а) рождается или умирает малый отрезок между двумя точками излома. В случае b) рождается или умирает гладкая дуга между двумя близкими точками одной гладкой ветви графика. В случае с) на графике на мгновение возникает особенность, близ которой он диффеоморфен кривой $x^{3}=y^{4}$, причем по одну сторону от этого значения параметра особенности (в этом месте) на графике нет, а по другую сторону график имеет обычный излом (как график функции $y=|x|$ ), малый при близком к критическому значении параметра.

Другими источниками особенностей оптимального среднего значения являются перестройки критических точек и значений целевой функции $f$. Типичная целевая функция имеет невырожденные («морсовские») максимумы и минимумы в некоторых отдельных точках фазовой окружности, причем все критические значения попарно различны. Если система зависит от параметра, то для типичных однопараметрических семейств систем при отдельных значениях параметра критическая точка целевой функции может вырождаться (как для функции $\left.f=1+x^{3}\right)$ или могут совпасть критические значения целевой функции в двух разных критических точках (как для функции $f=x^{4}-x^{2}$ ).

И, наконец, при отдельных значениях параметра возможны такие вырождения, когда и целевая функция, и задающие ограничения функции $r$ и $R$ сами по себе 
имеют лишь невырожденные особенности, но одна из критических точек одного типа (скажем, для $r$ ) оказывается критической и в другом смысле (скажем, для $f)$.

Рассмотрение каждого из перечисленных случаев несложно (по модулю уже известных в теории особенностей результатов - см., например, [2-5]). Теорема 1 получается сложной только из-за большого их числа.

Предположим, что параметр $p$ имеет значение $p_{0}$ общего положения, так что ни один из случаев а), b), с) не имеет места. Пусть $c-$ не критическое значение целевой функции $f$. Функции $r, R$ и $D=R-r$ имеют тогда простейшие «модульные» особенности (с простым разрывом первой производной от функции в некоторых специальных точках $x=\xi_{i}$, гладко зависящих от параметра $p$ вблизи ТОчКИ $\left.p_{0}\right)$.

ЛЕмма 1. Функиия «суммарная масса» распределения $\rho_{c}$,

$$
M(c)=\oint r(x) d x+\int_{f(x)>c} D(x) d x,
$$

является гладкой, за исключением отдельных точек с, где она имеет разрыв второй производной; в такой точке $c=f\left(\xi_{i}\right)$ (для одной из специиальньх для $D$ точек $\left.x=\xi_{i}\right)$.

Действительно, $d M / d c=-\sum_{j} D\left(x_{j}\right) /\left|f^{\prime}\left(x_{j}\right)\right|$, где $x_{j}-$ корни уравнения $f\left(x_{j}\right)=c$.

Если эти точки $x_{j}$ не специальны для $D$, то производная (как и точки $x_{j}$ ) гладко зависит от $c$. Если одна из точек $x_{j}$ совпадает со специальной точкой $\xi_{i}$, то $f\left(\xi_{i}\right)=c$, а первая производная $M$ по $c$ имеет в точке $c$ такую же «модульную» особенность, как функция $D$, поскольку $x_{j}$ гладко зависит от (некритического) значения $c$ целевой функции. Значит, сама функция $M$ имеет в этой точке $c$ простейший разрыв второй производной: при малых $|t|$

$$
M(c+t)=M(c)+a t+b_{ \pm} t^{2}+O\left(|t|^{3}\right),
$$

где $b_{+}>b_{-}$и $b_{+}$обслуживает область $t>0$, а $b_{-}-$область $t<0$.

ЛЕмма 2. При близких $\kappa p_{0}$ значениях параметра $p=p_{0}+s$ поведение функциии $M$ описывается подобной (3) формулой, в которой $c=f\left(\xi_{i}\right)$ гладко, как и $\xi_{i}$, зависит от малого параметра $s$, а значения коэффициентов $M(c)$, $a, b_{+}, b_{-}$все гладко зависят от малого параметра $s$.

Этот параметрический вариант леммы 1 доказывается так же, как эта лемма, приведенной выше формулой для $d M / d c$, учитывая гладкую зависимость особых точек $\xi_{i}$ функции $D$ и значений этой функции и ее односторонних производных по $x$ в этих особых точках от параметра $p$ вблизи его значения $p_{0}$.

Рассмотрим теперь немного более общую, чем $M$, функцию, выражающую интеграл от $f$ по распределению $\rho_{c}$ :

$$
N(c)=\oint f(x) r(x) d x+\int_{f(x)>c} f(x) R(x) d x .
$$

Для этой функции производная по $c$ выражается формулой

$$
\frac{d N}{d c}=-\sum_{j} \frac{f\left(x_{j}\right) D\left(x_{j}\right)}{\left|f^{\prime}\left(x_{j}\right)\right|}, \quad f\left(x_{j}\right)=c .
$$


Поэтому ее поведение вблизи специального значения $c=f\left(\xi_{i}\right)$ описывается аналогичной формуле (3) формулой (гладко, как и формула (3), зависящей от параметра $p$ вблизи $\left.p_{0}\right)$ с другими коэффициентами, описывающими, как и для $M$, простой разрыв второй производной $N$ по $c$.

Теперь мы исследуем с-среднее значение цзелевой функции,

$$
f_{c}=N(c) / M(c)
$$

максимум которого по $c$ и есть оптимальное среднее значение, $f_{*}$. Наша задача - исследовать зависимость этого максимума от значения параметра $p$. Мы предполагаем исследуемое семейство типичным.

ЛЕмма 3. Если для типичного значения $p_{0}$ параметра $р$ невырожденный максимум $f_{*}$ функциии $f_{c}$ достигается в неспецииальной точке $\left(c \neq f\left(\xi_{i}\right)\right)$, то этот максимум $f_{*}$ является (в окрестности точки $p_{0}$ ) гладкой функцией от параметра р. Если же максимум достигается в спецуиальной точке ( $c=$ $\left.f\left(\xi_{i}\right)\right)$, то функция $f_{*}$ испьтывает в точке $p=p_{0}$ простейший разрьв второй производной:

$$
f_{*}\left(p_{0}+s\right)=f_{*}\left(p_{0}\right)+A s+B_{ \pm} s^{2}+O\left(|s|^{3}\right),
$$

где не равные между собой числа $B_{+} u B_{-}$обслуживают $s>0$ u $s<0$ соответственно.

Заметим прежде всего, что $c$ - не критическое значение целевой функции $f$. Действительно, пусть $c-$ максимум целевой функции $f$. Тогда $M(c)=\oint r(x) d x$, $N(c)=\oint f(x) r(x) d x$. При небольшом уменьшении величины $c$ (на $t$ ) в распределении $\rho_{c}$ появится (вблизи точки максимума) отрезок длины порядка $\sqrt{t}$, где плотность $\rho$ увеличится на $D$. Следовательно, распределение масс сдвинется в сторону увеличения доли точек с бо́льшими значениями усредняемой функции $f$, поэтому среднее, $f_{c}$, возрастет. Значит, максимальное для $f$ значение с не является точкой максимума функции $f_{c}$ переменной $c$.

Если $c-$ минимум целевой функции, то

$$
M(c)=\oint R(x) d x, \quad N(c)=\oint f(x) R(x) d x .
$$

При увеличении величины $c$ на небольшое приращение $t$ плотность $\rho_{c}$ испытает уменьшение на отрезке длины порядка $\sqrt{t}$ вблизи точки минимума целевой функции. Среднее значение при этом возрастет, поскольку относительный вклад точек с меньшими значениями целевой функции уменьшится. Так что и минимальное для $f$ значение $c$ не является точкой максимума $c$-среднего.

ЗАмЕЧАНИЕ. Эти рассуждения - единственное место, где мы используем предположение о единственности точек максимума и минимума целевой функции. Случай, когда число локальных критических точек больше двух, нуждается в дополнительном исследовании. Но все остальные части наших рассуждений не зависят от числа критических точек (и результаты справедливы для некритических значений с даже и тогда, когда число критических точек больше двух).

Если в лемме 3 максимум достигается в неспециальной точке, то речь идет, согласно лемме 2, о максимуме гладкой функции, зависящей еще от параметра. Если максимум невырожден, то максимальное значение гладко зависит от параметра. Случай конкуренции нескольких локальных максимумов в этой ситуации исследуется, как при разборе перечисленных выше случаев а), b), с). 
Возникает разрыв первой производной глобального максимума по параметру: $f_{*}\left(p_{0}+t\right)=f_{*}\left(p_{0}\right)+A t+B_{ \pm} t+O\left(t^{2}\right)\left(B_{+}\right.$при $t \geqslant 0, B_{-}$при $\left.t \leqslant 0\right)$.

Если в лемме 3 максимум достигается в специальной точке, то формулы получаются такие: для $p=p_{0}+s$ имеют место разложения

$$
f_{c(s)+t}=f_{0}(s)+A(s) t+B_{ \pm}(s) t^{2}+O\left(|t|^{3}\right),
$$

где $c(s)=f\left(\xi_{i}(s)\right)$ - значение целевой функции в особой точке функции $D$ при $p=p_{0}+s$; коэффициенты $f_{0}, A, B_{ \pm}$- гладкие при малых $|s|$ функции, $B_{+} \neq B_{-}\left(B_{+}\right.$применяется при $t>0, B_{-}-$при $\left.t<0\right)$. Обе величины $B_{ \pm}(0)$ отрицательны, так как критическая точка - максимум при $s=0$.

При критическом значении параметра $p$ (когда $s=0$ ) максимум достигается при $t=0$, т.е. $A(0)=0$. При малом $s$ линейное по $t$ слагаемое имеет производную $A(s)$ порядка $s$. Поэтому критическая точка $t_{*}(s)$ функции $f_{c(s)+t}$ по $t$ будет определяться (в первом приближении по $s$ ) из условия $2 B_{ \pm} t_{*}+A=0$, откуда мы получаем асимптотику $t_{*}=q_{ \pm} s+O\left(s^{2}\right)$, где $q_{ \pm}=-A^{\prime}(0) / B_{ \pm}(0)$ (индексы коэффициентов $q$ и $B$ определяются знаком $t_{*}$, который меняется при изменении знака $s$, так как сами величины $q_{+}$и $q_{-}$, как и $B_{+}$и $B_{-}$, одного знака).

Подставляя найденное значение $t_{*}(s)$, мы приходим для максимального значения $c$-среднего (достигаемого в точке $\left.t_{*}(s)\right)$ к выражению

$$
f_{c(s)+t_{*}(s)}=f_{00}+f_{01} s+f_{02} s^{2}+C_{ \pm} s^{2}+O\left(|s|^{3}\right)
$$

(при малых $s$ ), что и доказывает лемму 3.

ЗАмечАНИЕ. Строго говоря, чтобы извлечь из этих лемм теорему 1 , нужно было бы рассмотреть еще оставшиеся исключенными случаи тех значений параметра $p$, при которых перестраиваются особенности функции $D$ (в соответствии с перестройками a), b), с), обсуждавшимися выше).

При этих значениях параметра $p$ на кривой $\{M(c), N(c)\}$ в некоторых местах $c_{*}$ возникают более сложные особенности, чем мы рассмотрели выше.

Но на поведение в зависимости от параметра $p$ оптимального среднего значения $f_{*}$ целевой функции эти необычные особенности кривой будут влиять только в том случае, когда указанная точка $c_{*}$ будет той самой точкой, где отношение $N / M$ максимально, т. е. точкой касания кривой $\{M, N\}$ с радиус-вектором.

Однако уже такое касание в обычной особой точке кривой, изученное в лемме 3, наблюдается лишь при некоторых исключительных значениях параметра $p$. Необычные особенности кривой появляются тоже при некоторых (исключительных в другом смысле) значениях $p$. Для семейства общего положения эти исключительные значения одного и другого рода различны. Поэтому перестройки особенностей функций $R$ и $r$ при изменении параметра $p$ не дают (для типичных семейств) новых особенностей зависимости оптимального среднего от параметра.

Остаются лишь «модульные» особенности (с простейшим разрывом первой производной), как для зависимости от параметра максимума типичной гладкой функции одной переменной, зависящей от одного параметра, и доставляемые леммой 3 особенности «типа $s|s|$ » с простейшим разрывом второй производной.

ЗАмечАниЕ. Прохождение особенностей типов а), b), с) функций максимума или минимума при изменении параметра приводит для зависимости от параметpa $s$ функций $M(c)$ и $N(c)$ к вполне определенным особенностям, имеющим следующие 3 вида. 
СлучАй а): гладкая зависимость от параметра нарушается (при одном из знаков его приращения, $s$ ) поправкой порядка $s^{2}$.

СлучАй b): гладкая зависимость от параметра нарушается (при одном из знаков его приращения, $s$ ) поправкой порядка $|s|^{3 / 2}$.

СлучАй с): гладкая зависимость от параметра нарушается (при одном из знаков его приращения, $s$ ) поправкой порядка $|s|^{7 / 2}$.

Последний результат получается из нормальной формы особенности «ласточного хвоста» (описывающей столкновение двух точек максимума),

$$
w=g+h x u+a s u^{2}-b u^{4} .
$$

Критические точки, $u$, определяются уравнением

$$
h x=4 b u^{3}-2 a s u,
$$

а критические значения - формулой

$$
w_{*}=g+3 b u^{4}-a s u^{2} .
$$

Столкновение происходит при нулевом значении параметра $s$ при $x=0$. Формулы (4), (5) (при фиксированном значении параметра $s$ ) задают на плоскости $\left\{\left(x, w_{*}\right)\right\}$ кривую, параметризованную критической точкой $u$. Эта кривая является графиком трехзначного (зависящего от $x$ ) критического значения функции $w$ от $u$.

Локальные максимумы соответствуют значениям $u$, расположенным левее первого и правее второго прохождения кривой через ее двойную точку (в которой $\left.x=0, u^{2}=a s /(2 b)\right)$. Теперь нетрудно оценить по формулам (4) и (5) изменение при изменении $s$ функции

$$
M_{+}=\int w_{*} d x=\int w_{*}(d x / d u) d u .
$$

Подставим в этот интеграл выражения (4) и (5) для $x$ и $w_{*}$. Приращение $M_{+}(s>0)-M_{+}(s<0)$ оказывается имеющим при малых $|s|$ величину порядка $|s| u_{*}^{5}$, т. е. порядка $|s|^{7 / 2}$. Такой же порядок величины имеет площадь криволинейного треугольника, ограниченного нашей кривой (между точками возврата и точкой самопересечения графика) при надлежащем знаке параметра $s$.

Таким путем, кроме теоремы 1, мы описываем и другие особенности зависимости кривых $\{M, N\}$ от $p$.

\section{§2. Смешанные стратегии}

Теперь мы рассмотрим ситуацию, в некотором смысле противоположную предыдущей: динамическая управляемая система будет иметь много положений равновесия, а задача будет состоять в том, чтобы между ними выбрать. Оптимальным часто оказывается, однако, не просто выбор одного из конкурирующих стационарных режимов, а динамическое блуждание между ними, в среднем дающее больший выигрыш, чем любой устойчивый равновесный режим.

Уравнение движения управляемой системы имеет прежний вид (1), но векторное поле $v$ имеет теперь (в произведении одномерного фазового пространства с координатой $x$ и одномерного пространства значений управляющего параметра с координатой $u$ ) целую кривую $K$ неподвижных точек: $v(x, u)=0$. 
Если вся фазовая траектория сводится или сходится к такой неподвижной точке, то временны́м средним значением целевой функции $f$ вдоль этой траектории будет значение целевой функции $f$ в самой этой точке. Поэтому для оптимизации временно́го среднего естественно прежде всего рассмотреть ограничение целевой функции $f$ на кривую равновесий, $K$, затем отыскать на $K$ точку максимума ограничения на $K$ целевой функции и, наконец, стремиться к этой точке приблизиться, выбирая управление.

Как мы сейчас увидим, при надлежащей стратегии управления можно приблизиться к доставляемому этой равновесной точкой условного максимума временно́му среднему значению. Поэтому мы перечислим (в теореме 2 ниже) типичные особенности «оптимальных» временны́х средних этого происхождения.

Однако стратегии их достижения вовсе не очевидны, так же, как не очевидно и отсутствие лучших стратегий.

В качестве примера можно рассмотреть систему $\dot{x}=u+x^{2}$ с параболической кривой $K$ неподвижных точек $\left(u=-x^{2}\right)$ и с целевой функцией $f=x$. В этом примере ни фазовое пространство, ни пространство значений управляющего параметра, $\{u\}$, не компактны, но в приведенных ниже общих рассуждениях я не буду заботиться об оговорках, необходимых для включения в общую теорию подобных некомпактных случаев (обычно достаточно наложить на $v$ граничные условия на бесконечности, препятствующие уходу фазовой точки туда за конечное время).

ОПРеДЕлЕНИЕ. Кривая $C$, пробегаемая на плоскости с координатами $(x, u)$, называется допустимой, если вдоль нее $d x>0$ там, где $v(x, u)>0$, и $d x<0$ там, где $v(x, u)<0$.

В приведенном примере: ниже параболы $u=-x^{2}$ надо двигаться влево, а выше - вправо (как это и делает динамическая система).

Для простоты мы будем еще предполагать, что не только на $K$, но и в окрестности кривой $K$ неподвижных точек допустимая кривая строго «вертикальна» $(d x \equiv 0)$.

ПрЕДЛОЖеНИЕ. Для любой допустимой замкнутой кривой существует такое управление $u=U(t)$, что точка $(x(U(t)), u=U(t))$ периодически движется вдоль этой допустимой кривой.

ПримеР. Для уравнения $\dot{x}=u+x^{2}$ в качестве допустимой кривой возьмем прямоугольник (углы которого можно было бы еще скруглить) со сторонами $x=$ $A, x=B$ и $U=a, U=-b$ (считая, скажем, $0<A<B, a>0, b>B^{2}$ ).

Управление $U(t)$ можно здесь выбрать в виде кусочно-постоянной разрывной функции со значениями $a$ и $-b$. В качестве начальной точки допустимой кривой выберем точку $(x=A, U=a)$. Значение $U=a$ сохраняется до тех пор, пока $x$ не дорастет до $B$, после чего выбираем $U=-b$ и оставляем его равным $-b$, пока $x$ не уменьшится до $A$. И потом все повторяется периодически.

Построение управления по любой допустимой кривой $C$ основано на такой же конструкции. Скажем, если точка $(x, u)$ кривой $C$ принадлежит области $v>0$ и в этой точке $d u>0$, то сойдем с кривой $C$, немного увеличив $u$, и немного подождем, пока движение со скоростью $v$ не вернет фазовую точку на $C$, а затем будем повторять этот процесс, пока не получим приближающуюся к $C$ ступенчатую ломаную. Переход к пределу бесконечно малых шагов не вызывает затруднений. 
Если $A$ и $B$ близки к некоторому общему значению $X$ фазовой координаты $x$, то временно́е среднее целевой функции $f$ вдоль допустимой кривой, заключенной в полосе $A<x<B$, будет близким к значению $f(X)$.

Таким образом, мы построили специальные управления, для которых временны́е средние значения целевой функции оказываются почти равными значению самой этой функции в выбранной произвольно точке на кривой равновесий, $K$.

ЗАмЕчАНИЕ. Некоторые точки кривой равновесий являются притягивающими для соответствующего постоянного значения параметра управления, $u$ (например, таковы точки $K$ в левой полуплоскости $(x<0)$ в примере $\left.\dot{x}=u+x^{2}\right)$. Построить управление $U$, приводящее фазовую точку к такой притягивающей точке, нетрудно. В этом случае временно́е среднее значение, очевидно, будет равно значению целевой функции в этой притягивающей точке.

Но допустимые кривые определяют и гораздо более сложные «допустимые стратегии», позволяющие приблизиться к максимуму ограничения целевой функции на кривую неподвижных точек управляемой динамической системы, что уже вовсе не так легко сделать, если точка максимума - не притягивающая.

Мы получили, таким образом, семейство допустимых стратегий, реализующее в качестве оптимального для этого класса стратегий временно́го среднего значения максимум ограничения целевой функции на кривую неподвижных точек управляемой динамики. Легко доказывается

Теорема 2. Максимум $F(p)$ ограничения гладкой функции $f$ на кривую неподвижных точек $\left(K_{p}=\{x, u: v(x, u ; p)=0\}\right)$ гладкой динамической управляемой системь $\dot{x}=v(x, u ; p)$, гладко зависящей от параметра $p$, является гладкой функцией от $p$, исключая дискретно лежащие особые точки, в окрестности каждой из которых график функции $F$ диффеоморфен графику одной из двух функций $F_{1}=|p|, F_{2}=\{0$ при $p<0,1+\sqrt{p}$ при $p>0\}$ (для $f$ u $v$ общего положения).

Пусть кривая положений равновесия управляемой системы при некотором значении параметра $p$ неособа (так что $0-$ не критическое значение функции $v$ ). Тогда наши специальные допустимые стратегии доставляют среднее значение целевой функции, равное максимуму типичной гладкой функции двух переменных по одной из двух переменных:

$$
F(p)=\max _{K_{p}} f(x)=\max _{s \in S^{1}} G(s, t), \quad t=p-p_{0} .
$$

Как и в случае максимума функции $w$ по $u$, рассмотренном в 1 , график функции $F$ имеет такие же особенности, как линия горизонта типичного ландшафта $G$ : для некоторых $a>b$ при малых $t$ выполняются соотношения

$$
\begin{aligned}
& F\left(p_{0}+t\right)=c+a t+O\left(t^{2}\right) \quad \text { при } t \geqslant 0, \\
& F\left(p_{0}+t\right)=c+b t+O\left(t^{2}\right) \quad \text { при } t \leqslant 0 .
\end{aligned}
$$

Это - первый случай теоремы 2 («модульная особенность»).

Если же кривая положений равновесия, $K_{p_{0}}$, имеет особенность, то для семейства общего положения при прохождении параметром $p$ значения $p_{0}$ эта кривая испытывает морсовскую перестройку (типичные примеры: $v=x^{2} \pm u^{2}+p, p_{0}=0$ ).

В этом случае при прохождении параметром $p$ критического значения по одну сторону от него появляется новый отрезок значений $x$ (а значит, и $f(x)$ ) на 
кривой $K_{p}$, а именно отрезок длиной порядка $\sqrt{|t|}$. Если значение $F_{0}$ функции $f(x)$ в перестраивающейся критической точке больше, чем на остальной части кривой неподвижных точек, то (по одну сторону от значения $t=0$ ) появляются максимальные значения $F(t)=F_{0}+F_{1} \sqrt{|t|}+O(t)$.

Это - второй случай теоремы 2 (по другую сторону от $t=0$ функция $F$ гладкая и меньше $F_{0}$; диффеоморфизм плоскости, приводящий ее график к указанной в теореме нормальной форме, получается переменным сдвигом вдоль оси значений на плоскости графика, превращающим гладкую часть графика в горизонтальную прямую, и затем - гладко зависящим от горизонтальной координаты растяжением вертикальной координаты, нормирующим ее).

Здесь мы для простоты по-прежнему предполагали, что $x \in S^{1}, u \in S^{1}$. Но более общие случаи (например, с любым компактным фазовым многообразием) не представляют новых трудностей, особенно - если размерности пространства управляющих переменных, $\{u\}$, и пространства параметров, $\{p\}$, невелики.

Но даже в нашем простейшем случае, когда оба эти пространства одномерны, теорема 2 описывает особенности лишь тех временны́х средних, которые получаются при использовании стратегий, оптимальных среди наших специальных допустимых стратегий. Будут ли такими же особенности зависимости от параметра для по-настоящему оптимальных временны́х средних, я не знаю.

Эти последние особенности было бы интересно сравнить с особенностями функций максимума типичных семейств функций от любого (или даже от бесконечно большого) числа переменных (для типичных семейств функций, зависящих от такого же числа параметров, как и рассматриваемая в семействе управляемая динамическая система).

Например, функции максимума типичных семейств функций на фиксированном многообразии топологически эквивалентны морсовским гладким функциям. А так ли это для максимальных значений временны́х средних по всем управлениям в типичных семействах управляемых систем, неизвестно.

Вопросы о типичных фазовых переходах, т.е. об особенностях зависимости максимальных значений средних величин от параметров задачи, интересны также для систем уравнений с частными производными, в которых роль времени, $t$, по которому происходит усреднение, играет точка интегрального многообразия, размерность которого больше единицы. При этом целевую функцию естественно считать зависящей не только от искомых «значений полей» (играющих роль значений $x$ для точек «орбиты» $\{(x(t))\}$ в рассмотренном выше случае одномерного времени), но и от их частных производных $\partial x_{i} / \partial t_{j}$, да и от значений используемых управляющих параметров, $u_{k}$. Уже в нашей одномерной задаче $(1)$ можно было бы рассматривать более общую целевую функцию $f(x, u)$ вместо $f(x)$, почти не меняя ни результатов, ни доказательств.

В качестве примера задач с «многомерным временем» можно рассмотреть возникающие в псевдопериодической топологии случаи «квазипериодических» функций или псевдопериодических сумм линейных функций с периодическими функциями. Рассмотрим периодическую функцию (например, тригонометрический многочлен) от $n$ переменных и ограничим ее на играющую роль интегрального многообразия «иррациональную» плоскость размерности $k$ в $n$-мерном аффинном пространстве (являющемся универсальной накрывающей тора, где исходная периодическая функция на самом деле определена). Средние различных 
аналитических и топологических характеристик таких «квазипериодческих» ограничений недавно изучены С. М. Гусейн-Заде (см. [6, 7]). Фазовые переходы должны здесь появляться в виде особенностей зависимости этих средних от параметров (например, от коэффициентов исходного тригонометрического многочлена и от выбора аффинной «интегральной плоскости»), но они мало изучены, хотя вопрос об их изучении давно известен и обсуждался, например, в работе [8], продолженной в $[7,6]$.

Квазипериодический аналог теоремы Харнака оценивает сверху как топологические, так и метрические средние характеристики псевдопериодических функций и многообразий (через степень тригонометрического многочлена или через диаграмму Ньютона той периодической функции, ограничение которой на иррациональную плоскость задает исследуемый квазипериодический объект). Длина кривой (на стандартного размера торе), где обращается в нуль тригонометрический многочлен степени $n$, ограничена сверху константой, не зависящей от коэффициентов многочлена, а зависящей только от $n$. Ограничены сверху и длины всех алгебраических кривых фиксированной степени на сфере радиуса единица в трехмерном пространстве. Подобные аналоги имеет и теорема Штурма-Гурвица о нулях рядов Фурье: длина линии на стандартном торе, где равен нулю тригонометрический многочлен, ограничена снизу некоторой (не зависящей от коэффициентов многочлена) постоянной $c(n)$, если этот многочлен ортогонален всем тригонометрическим многочленам степени меньше $n$.

\section{ЛИТЕРАТУРА}

1. Арнольд В. И. Выпуклые оболочки и повышение производительности систем при пульсирующей загрузке. Сиб. матем. журнал, 28, № 4, 27-31 (1987).

2. Арнольд В. И., Варченко А. Н., Гусейн-Заде С. М. Особенности дифференцируемых отображений, I. Наука, М., 1982.

3. Арнольд В. И., Васильев В. А., Горюнов В. В., Ляшко О. В. Особенности І. Локальная и глобальная теория. Динамические системы-6. Итоги науки и техники. Современные проблемы математики. Фундаментальные направления, т. 6, ВИНИТИ, М., 1988.

4. Арнольд В. И., Васильев В. А., Горюнов В. В., Ляшко О. В. Особенности II. Классификация и приложения. Динамические системы-8. Итоги науки и техники. Современные проблемы математики. Фундаментальные направления, т. 39, ВИНИТИ, М., 1989.

5. Арнольд В. И. Теория катастроф. Наука, М., 1990.

6. Gusein-Zade S. M. On the topology of quasiperiodic functions. In: Pseudoperiodic Topology (V. Arnold, M. Kontsevich, and A. Zorich, eds.). Amer. Math. Soc. Transl., Ser. 2, Vol. 197, 1999, pp. 1-8.

7. Гусейн-Заде С. М. Число критических точек квазипериодического потенциала. Функц. анализ и его прил., 23, вып. 2, 55-56 (1989).

8. Arnold V. I. Remarks on quasicrystallic symmetries. Phys. D, 33, 21-25 (1988).

Математический институт им. В. А. Стеклова, CEREMADE, Universitè Paris-Dauphine

Поступило в редакцию 14 ноября 2001 г. 